highly significant. The statistical analysis did not show any evidence of a curvilinear or logarithmic relation over the dose range studied, although we too would have expected this.

This was a long term study with each of the four treatment periods being of two months' duration, thus showing a long term effect dismissed by Drs Davidson and Cooper. The improvement in exercise tolerance in many of our subjects was clinically extremely useful, and perhaps the greatest testimony to this was the problems we encountered in controlling patients' symptoms during what retrospectively turned out to be the placebo periods. It is also relevant that all but seven of our original 38 patients opted to continue receiving long term theophylline treatment at medium or high dose at the end of the study.

The major thrust of our work, however, was that changes in forced expiratory volume in one second and peak expiratory flow rate poorly reflect symptomatic change in this group of patients and that to the extent that such indices have been used in this subject of research reassessment of such research methods is required. While we agree that theophylline is probably not "uniquely useful" in this context, it is of value in many patients if used with care. Indeed all our published work in this subject has attempted to promote the proper rather than the indiscriminate use of this compound.

M D PEAKE H CHRYSTYN

Pontefract General Infirmary,

Pontefract WF8 1PL

B A MULLEY

School of Pharmacy, University of Bradford,

Bradford BD7 1DP

1 Rodenstein DO, Stanescu DC. Reassessment of lung volum measurement by helium dilution and by body plethysmography in chronic airflow obstruction. Am Rev Respir Dis 1982;126: 1040-4.

\section{Psychosis from alcohol or drug abuse}

SIR,-Drs M Lipsedge and R Littlewood doubt the finding of Drs Cohen and Johnson that the psychotic symptoms of many patients admitted to psychiatric wards are caused by drug or alcoho abuse,,$^{2}$ but this was my own finding as ward psychiatrist at San Francisco General Hospital during the explosion of drug abuse in the late 1960s. Unless I knew patients from previous admissions I did not order regular drug treatment until I had observed them for a few days. By then many had lost their symptoms and could be discharged. Some acknowledged that they had taken drugs. I did authorise the use of antipsychotic drugs if required to control agitation, but these were often not needed or else needed in amounts too small to account for the resolution of symptoms Perhaps their being in the safe environment of a hospital was enough to allow most of these patients to be cared for with little or no medication.

When people admitted with psychotic symptoms are immediately started on drug treatment their recovery, which in many cases would occur withou such drugs, is usually taken as confirmation that they are schizophrenic. Once applied this label usually sticks. One result is years of unnecessary medication with its hazards, and another is the creation of a class of misdiagnosed people who are supported at public expense and who in many cases use their payments to keep on buying drugs.

San Francisco, California 94131

DONALD GALFOND United States

1 Lipsedge M, Littlewood R. Psychosis from alcohol or dru abuse. BrMed f 1988;297:1612. (17 December.)

2 Cohen SI, Johnson K. Psychosis from drug or alcohol abuse. Br Med f 1988;297:1270-1. (12 November.)

\section{"There is a tide in the affairs of women ..."}

SIR, - We were fascinated by the paper of Professor G V P Chamberlain and Mr M F Asam ${ }^{1}$ but surprised that they did not find results that agreed with the shepherd who had the immense practical experience of 50 years. This may be due to the distance of the delivery suite at St George's Hospital from the River Thames. The delivery suite of St Thomas's Hospital must be the closest unit to the Thames, being on the embankment less than 20 metres from the water's edge. In view of this we thought that a similar study should be carried out there.

We used the same time span as that of the authors. During July and August 1987, 416 women delivered spontaneously. We used the high tide times at Westminster Bridge in Greenwich mean time.

Our results showed that there was a significant difference between the number of women delivering around the flood and the number delivering at the time of the ebb tide (table), with more women delivering around the flood tide $\left(\chi^{2}\right.$ test; $\left.\mathrm{p}<0.025\right)$.

Number of women giving birth in relation to time of high tide at St Thomas's Hospital

$\begin{array}{lllllll}\text { Time (h) from high tide: } & -6 & -4 & -2 & +2 & +4 & +6 \\ \text { No of women: } & 64 & 68 & 88 & 81 & 65 & 52\end{array}$
No of women

We conclude that the shepherd's memory served him well and that it is the distance to the ebb and flood tide that is important. Perhaps people do, after all, behave like sheep.

D RAJASINGHAM

L P MARSON

A E MILLS

M DOOLEY

Department of Gynaecology,

St Thomas's Hospital Medical School,

London SE1 7EH

1 Chamberlain GVP, Azam MF. A time to be born. Br Med $\mathcal{J}$ 1988;297:1637. (24-31 December.)

\section{A national ethics committee}

SIR, - We welcome the editorial of Dame Mary Warnock on the need for a national ethics committee. ${ }^{1}$ It is important that a national committee should promote discussion and offer guidance on the major ethical issues raised by new subjects of treatment and research. In addition, the committee could give advice on detailed issues in the conduct of research, such as the appropriate means for ensuring that pregnant women are not subjects and the provision of compensation arrangements, points on which there is substantial variation in the approach of both drug companies and local ethical committees.

The conduct of research should, however, also be the responsibility of local ethical committees as at present. Local circumstances have a bearing on the ethics of research protocols. Issues such as the availability of appropriate multilingual advice for subjects and the balancing of demands on scarce resources such as nursing and pharmacy are matters with ethical connotations that require local consideration. For these reasons we oppose the view that a national ethics committee should consider all multicentre trials ${ }^{2}$ and we are pleased to note that this proposal is not included in Mary Warnock's call for a national body.

Our experience of the working of local ethical committees, partly gathered from a seminar for lay members, ${ }^{3}$ confirms Mary Warnock's suggestion that their competence varies greatly. Her proposal for a national committee might well be coupled with action to improve the functioning of local committees. The collaboration of effective local committees with the national committee should be an important feature of the proposed reform.

P MOODIE T MARSHALL

Faculty of Law and Department of Social Medicine.

University of Birmingham,

Birmingham B15 2TJ

1 Warnock M. A national ethics committee, Br Med f 1988:297: 1626-7. (24-31 December.)

2 Nott PN, Steel EA. Scrutinising research ethics committees. Br Med F 1988;297:1333.

3 Marshall T, Moodie P. Scrutinising research ethics committees. BrMed J 1988;297:753.

\section{Are all infants of diabetic mothers "macrosomic"?}

SIR,-Dr R J Bradley and colleagues recently presented data purporting to show a unimodal right sided shift of birth weight of diabetic mothers compared with a singleton reference population and suggested that all infants of diabetic mothers are growth promoted to some degree by the maternal disease.' The report, however, raises several important questions regarding methodology and ignores the wider context of fetal macrosomia or "big babies" previously discussed in an editorial. ${ }^{2}$

Classically, maternal diabetes has been linked to large babies, but several studies have suggested that most macrosomic babies are born to mothers who have no evidence of carbohydrate intolerance. Between 1978 and 1982, 6530 babies weighing $3500-4500 \mathrm{~g}$ and 257 weighing $>4500 \mathrm{~g}$ were born at the Royal Maternity Hospital, Belfast. Of those weighing $>4500 \mathrm{~g}$, only five were born to mothers with established insulin dependent diabetes, six to mothers with results indicating diabetes on glucose tolerance testing, and seven to mothers with impaired glucose tolerance during the pregnancy.

Most factors linked with macrosomia are poor predictors, ${ }^{2}$ their presence only doubling the risk. Nevertheless, the factors which are the main determinants of birth weight include sex, parity, the birth of a previous small or large gestational baby, and smoking during pregnancy. ${ }^{3}$ Dr Bradley and colleagues refer only to maternal height and weight in a singleton population, give few data about the matching of controls, and make the critical assumption of uniformity in estimating gestational age.

Maternal hyperglycaemia stimulates fetal insulin production, which is recognised as one of the main fetal growth factors. To what extent lesser degrees of hyperglycaemia may contribute to increased birth weight is still uncertain. Improved glycaemic control in pregnancy has reduced the number of large babies of insulin dependent diabetic mothers, and recognition of lesser degrees of impaired glucose tolerance may permit both obstetric and metabolic intervention. Though Dr Bradley and colleagues emphasise the importance of recognising glycaemic control as a continuum, no information is given about the degree of control during pregnancy or before conception, nor are details of any correlation between maternal blood glucose concentration and fetal birth weight given. It is also questionable whether combining a population of patients with pre-existing diabetes and gestational diabetes, in which the pathogenesis is different, is appropriate.

We have recently reported a diabetic pregnancy in which despite impeccable glycaemic control throughout a typical macrosomic infant was born weighing $5092 \mathrm{~g}$. ${ }^{+}$The use of the term macrosomia in its proper descriptive sense is commendable whether in a diabetic or non-diabetic context. Rather than engendering the concept of an all or none phenomenon, the term simply emphasises 